\title{
ETS-1 Expression Is Hypoxia-independent in Glioblastoma- derived Endothelial and Mesenchymal Stem-like Cells
}

\author{
DOMINIK KOESSINGER ${ }^{1,2}$, VALERIE ALBRECHT ${ }^{1}$, FLORIAN FABER ${ }^{1}$, \\ IRENE JAEHNERT $^{1}$ and CHRISTIAN SCHICHOR ${ }^{1}$ \\ ${ }^{1}$ Department of Neurosurgery, Ludwig Maximilians University of Munich, Munich, Germany; \\ ${ }^{2}$ Department of Neurosurgery, Wuerzburg University Hospital, Wuerzburg, Germany
}

\begin{abstract}
Background: Tumor cells infiltrating the brain are a typical hallmark of glioblastoma. Invasiveness of glioma cells has been associated with ETS proto-oncogene 1 (ETS1). In non-glial tumors, ETS-1 expression has been linked to hypoxia. However, it is not known whether hypoxia regulates ETS-1 expression in glioblastoma. Materials and Methods: The spatial distribution of ETS-1 expression in primary glioblastoma was assessed using immunohistochemistry. ETS1 expression in glioblastoma-derived mesenchymal stem-like cells (gbMSLCs) was determined using immunocytochemistry. The effect of hypoxia on ETS-1 expression of gbMSLCs, glioma cell lines and glioblastoma-derived endothelial cells was assessed using polymerase chain reaction and immunoblotting. Results: Our immunohistochemical studies revealed ETS-1 expression in stromal and endothelial glioblastoma cells. Stromal ETS-1 expression in glioblastoma correlated with microvessel density. gbMSLCs were found to express ETS-1. In all examined cell lines, ETS-1 transcription and expression were independent of hypoxia. Conclusion: In glioblastoma, ETS-1-expression is not dependent on hypoxia, but correlates with tumor vascularization.
\end{abstract}

Primary glioblastoma (GBM) is the most common and most malignant primary brain tumor $(1,2)$. Its infiltrative growth pattern with diffuse spread of cancer cells in healthy adjacent brain tissue mainly contributes to its poor therapeutic response, resulting in a median survival of only 15 months (1). Furthermore, the inevitable subsequent failure of the current treatment regimen, consisting of surgical resection

Correspondence to: PD Dr. Christian Schichor, Department of Neurosurgery, Ludwig Maximilian University of Munich, Marchioninistrasse 15, D-81377 Munich, Germany. Tel: +49 89440072581, Fax: +49 89440072592,e-mail: christian.schichor@ med.uni-muenchen.de

Key Words: ETS-1, primary glioblastoma, hypoxia, mesenchymal stem cell properties, tumor endothelial cells. with adjuvant radio- and chemotherapy, is attributed to a stem-like tumorigenic subpopulation of cells that are capable of self-renewal and display multiple different subclones (3).

ETS proto-oncogene 1 (ETS-1) is a transcription factor that is involved in differentiation of hematopoetic cells and angiogenesis (4-8). ETS-1 also plays a crucial role in carcinogenesis. As a proto-oncogenic factor, ETS-1 is capable of activating genes associated with angiogenesis, metastasis and invasive behavior in multiple tumor types (911). Additionally, ETS-1 expression correlates with microvessel density in some non-glial tumors and is an independent negative prognostic marker in different tumor entities such as breast, ovarian, pancreatic and colorectal cancer $(9,12-14)$. Likewise, ETS-1 has been shown to positively correlate with grading in astrocytic tumors (15).

Previous descriptions of the spatial expression pattern of ETS-1 in GBM are contradictory. Kitange et al. described positive immunoreactivity for ETS-1 in $65 \%$ of GBMs identifying a homogenous, mainly nuclear, partly cytosolic ETS-1 staining in 10-90\% of tumor cells as well as in endothelial cells of small tumor vessels (15). Differing from that, Valter et al. found ETS-1 expression in glioma to be restricted only to endothelial cells of tumor microvessels, especially in areas of presumably active neoangiogenesis (16).

In glioma cells, ETS-1 has been demonstrated to increase motility and invasiveness by up-regulating urokinase-type plasminogen activator (UPA) and promoting cell adhesion by enhancing the expression of fibronectin $\alpha 5(17,18)$. Using the rat glioma cell line $\mathrm{C} 6$, Sahin et al. indicated that functional inhibition of ETS-1 reduces cell proliferation, migration and tumor growth in vitro and in vivo (19).

However, possible mechanisms leading to the up-regulation of ETS-1 in glioma have not yet been elucidated. In urinary transitional carcinoma cells, hypoxia induces ETS- 1 mRNA transcription and protein expression mediated by hypoxiainducible factor 1 (HIF1) (20). Further evidence in the literature indicates a dependence of ETS-1 expression on hypoxia in hepatocellular carcinoma (21). Contrary to these findings, Qiao et al. demonstrated that hypoxia transiently 
increases ETS-1-mRNA transcription in pancreatic adenoma cells, while the ETS-1 protein level decreases (22). This implies that the impact of hypoxia on ETS-1 expression varies in different tissues.

GBM are rapidly-growing tumors with characteristic hypoxic conditions resulting in intratumoral necrosis (23). Therefore, it is reasonable to hypothesize that ETS-1 expression in GBM might be driven by hypoxia.

In our study, we aimed to further characterize the spatial ETS-1-expression pattern, especially regarding tumor vascularization. Subsequently, we investigated the dependence of ETS-1 expression on hypoxia in different GBM cell lines, GBM-derived mesenchymal stem-like cells (gbMSLC) as well as GBM-derived primary endothelial cells (TUEC).

\section{Materials and Methods}

Tissue collection. The tumor tissue collection was approved by the local Ethics Committee (project number 325-11). GBM tissue was retrieved from patients who underwent tumor resection in our clinic. Written informed consent was obtained prior to surgery.

Bone marrow aspirate from a healthy donor, not further needed for transplantation, was obtained from Aktion Knochenmarkspende Bayern e.V., Gauting, Germany after written informed consent.

Isolation of primary mesenchymal stem-like cells (bmMSC) from bone marrow. The isolation protocol has been published elsewhere (24). Briefly, the mononuclear cell fraction was isolated using Ficoll density gradient centrifugation (Amersham plc, Amersham, UK). Cells were cultured in Dulbecco's modified Eagle's medium (DMEM) (Biochrom, Berlin, Germany), 2mM glutamine, 100U/ml penicillin, $0.1 \mathrm{mg} / \mathrm{ml}$ streptomycin (Gibco, Carlsbad, CA, USA), $20 \%$ fetal bovine serum (FBS) (Biochrom) at $37^{\circ} \mathrm{C}$ in $5 \% \mathrm{CO}_{2}$. Cells were examined by flow cytometry and stained positively for CD29, CD44, CD90, CD105 and CD146 and negative for CD45 and CD34 (data not shown).

Isolation of gbMSLC from primary GBM tissue. The method has been published elsewhere (25). Fresh GBM tissue was obtained from the operating theater, minced, homogenized and incubated with collagenase/dispase (final concentration $1 \mathrm{mg} / \mathrm{ml}$; Roche, Basel, Switzerland) for $2 \mathrm{~h}$ at $37^{\circ} \mathrm{C}$. The cell solution was then filtered by a $30 \mu \mathrm{m}$ nylon mesh (Miltenyi, Bergisch Gladbach, Germany). The mononuclear cell fraction was isolated using Ficoll density gradient centrifugation (Amersham). The CD133-positive cell population was depleted using CD133 microbead kit following the manufacturer's protocol (Miltenyi). Cells were cultured in DMEM (Biochrom), $2 \mathrm{mM}$ glutamine, $100 \mathrm{U} / \mathrm{ml}$ penicillin, 0.1 $\mathrm{mg} / \mathrm{ml}$ streptomycin (Gibco), $20 \% \mathrm{FBS}$ (Biochrom) at $37^{\circ} \mathrm{C}$ in $5 \%$ $\mathrm{CO}_{2}$. Cells were examined by flow cytometry and stained positively for the mesenchymal progenitor markers CD29, CD44, CD90 and CD105 and negative for CD34 (data not shown).

Isolation of endothelial cells (TUEC) from primary GBM. Tumor tissue was received and homogenized as described above. The cells were processed in M199 medium (LONZA, Basel, Switzerland), $10 \%$ FBS (Biochrom), $100 \mathrm{U} / \mathrm{ml}$ penicillin, $0.1 \mathrm{mg} / \mathrm{ml}$ streptomycin (Gibco). For endothelial cell isolation, Dynabeads (ThermoFisher,
Darmstadt, Germany), coated with antibodies for both CD31 (Serotec, Duesseldorf, Germany) and CD34 (DAKO, Glostrup, Denmark) using magnetic particle sorter were used. Cells were cultured in microvascular endothelial cell growth medium, FCS-Kit, advanced (Provitro, Berlin, Germany) on gelatin at $37^{\circ} \mathrm{C}$ in $5 \% \mathrm{CO}_{2}$.

Cell lines. The human glioma cell lines U87MG, U251 and U373 (ATCC, Manassas, VA, USA) and the endothelial cell line human umbilical vein endothelial cells (HUVECs) (Provitro) were cultured in DMEM (Biochrom), $2 \mathrm{mM}$ glutamine, $100 \mathrm{U} / \mathrm{ml}$ penicillin, 0.1 $\mathrm{mg} / \mathrm{ml}$ streptomycin (Gibco), 10\% FBS (Biochrom) or microvascular endothelial cell growth medium, FCS-Kit, advanced (Provitro), respectively at $37^{\circ} \mathrm{C}$ in $5 \% \mathrm{CO}_{2}$.

Hypoxia assays. Glioma and endothelial cells were seeded at a density of $1 \times 10^{6}$, bmMSCs and gbMSLCs at a density of $5 \times 10^{5}$ cells per $75 \mathrm{~cm}^{2}$ flask, respectively. After $24 \mathrm{~h}$, the media were removed, cells washed twice with $\mathrm{PBS}$, then corresponding media without FBS or growth factor supplements were added. Cells were subsequently incubated either under normoxic $\left(21 \% \mathrm{O}_{2}\right)$ or hypoxic conditions $\left(0.1 \% \mathrm{O}_{2}\right)$ at $37^{\circ} \mathrm{C}$ in $5 \% \mathrm{CO}_{2}$ for $48 \mathrm{~h}$ before being harvested for mRNA or protein isolation.

$m R N A$ isolation and RT-PCR. mRNA from commercial glioma cell lines, gbMSLCs, bmMSCs and endothelial cells was isolated using the RNEasy Mini Kit (Qiagen, Hilden, Germany) and cDNA transcription was conducted using QuantiTect Reverse Transcription-Kit (Qiagen) according to the manufacturer's instructions. PCR was performed using Hot Start Taq Master Mix Kit (Qiagen). The following oligonucleotide sequences were used: ETS-1 fwd: 5'-GTT-AAT-GGA-GTC-AACCCA-GC-3', ETS-1 rev: 5'-GGG-TAG-CGA-CTT-CTT GTT-TG-3', Glyceraldehyde 3-phosphate dehydrogenase $(G A P D H)$ fwd: 5'-TCCAAA-ATC-AAG-TGG-GGC-GAT-GCT-3', GAPDH rev: 5'-ACCACC-TGG-TGC-TCA-GTG-TAG-CCC-3'. PCR products were analyzed using agarose gel electrophoresis and visualized using ethidium bromide staining. PCR product size was 274 bp for ETS-1 and 574 bp for GAPDH. The size of amplification products was determined by a 50 -bp DNA ladder $(0.1 \mu \mathrm{g} / \mu \mathrm{l}$ Gene Ruler 50 -bp DNA ladder; Fermentas, St. Leon-Rot, Germany). Gels were photographed using a digital gel documentation system (Serva, Heidelberg, Germany) and quantified using Image (NIH, Bethesda, MD, USA) (26). ETS-1 expression was normalized using $G A P D H$ expression.

Protein extraction and western blot analysis. Commercial glioma cell lines, gbMSLCs, bmMSCs and endothelial cells were detached using a cell scraper in cold lysis buffer $(10 \mathrm{mM}$ Tris, $0.5 \%$ NP-40, $150 \mathrm{mM} \mathrm{NaCl}, 10 \%$ glycerol, $2 \mathrm{mM}$ EDTA, $1 \mathrm{mM}$ dithiothreitol, $0.5 \mathrm{mM}$ phenylmethylsulfonyl fluoride, pH8.0) with protease inhibitor cocktail (Sigma-Aldrich St. Louis, MO, USA) and lysed by passing through a $26-\mathrm{G}$ needle several times. Cellular debris was removed by centrifugation. Protein concentration was determined using Quickstart Bradford Kit 2 (Bio-Rad, Hercules, CA, USA). Thirty micrograms of total protein per sample were subjected to sodium dodecyl sulfate-polyacrylamide gel electrophoresis (SDSPAGE) with a $4 \%$ polyacrylamide stacking gel and $7.5 \%$ polyacrylamide running gel in Tris-glycine buffer $(\mathrm{pH} 8.3)$ before being transferred to Immun-Blot polyvinylidene difluoride membranes (Bio-Rad). Membranes were incubated with Roti blocking solution (Carl-Roth, Karlsruhe, Germany) for $1 \mathrm{~h}$ at room temperature and subsequently incubated with primary antibody 
against ETS-1 (1:1,000; Millipore, Darmstadt, Germany) and secondary antibody (Immun-Star Goat Anti-Mouse (GAM)horseradish peroxidase (HRP); 1:10,000; BioRad). HRP-conjugated antibody against $\beta$-actin $(1: 20,000$; Santa Cruz, Dallas, TX, USA) was used as an internal control. Signals were visualized using Immun-Star HRP Chemiluminescent Kit (Bio-Rad) and lightsensitive imaging film (Super X-Ray RX; Fuji, Grangemouth, UK). Blots were scanned and quantified using ImageJ. ETS-1 expression was normalized using $\beta$-actin expression.

Immunohistochemistry/immunocytochemistry. For tissue fixation, HOPE starter kit was used according to the manufacturer's instructions (Innovative Diagnostik Systeme, Hamburg, Germany). Tumors were stained for ETS-1 (1:1500; Santa Cruz), CD31 (M0823, 1:100, Dako), mutant IDH (DIA-H09: Anti-IDH1 R132H, 1:100, Dianova), followed by DAB staining (K3466, Dako). Glioma cell lines, gbMSLCs and bmMSC were seeded on glass coverslips and incubated for $24 \mathrm{~h}$ at $37^{\circ} \mathrm{C}$ to adhere. Cells were fixed with $4 \%$ paraformaldehyde, permeabilized with NP-40 (Roche) and stained for ETS-1 (1:2000; Santa Cruz), followed by 3-amino-9ethylcarbazole staining (Dako). Cell nuclei were counterstained with hematoxylin (Merck, Darmstadt, Germany).

Statistical analysis. Statistical analysis was conducted using GraphPad Prism 7.02 (Graphpad Software, La Jolla, CA, USA). pValues were calculated using Mann-Whitney $U$-test. $p$-Values below 0.05 were deemed to be significant.

\section{Results}

Expression and local distribution of ETS-1 in human glioma tissue. For examination, three primary GBM were chosen, from which cells with mesenchymal stem cell properties (gbMSLCs) were isolated. In hematoxylin and eosin staining, all tumors showed classical histological features of grade IV GBM, such as cellular pleomorphism, nuclear atypia with high mitotic activity, distinct necrotic areas and microvascular proliferation (Figure 1a). No immunoreactivity for mutant isocitrate dehydrogenase 1 (IDH1) was observed (Figure 1b).

All tumor specimens displayed specific perinuclear immunoreactivity for ETS-1 in both tumor cells and intratumoral endothelial cells (Figure 1c). To determine the spatial correlation of ETS-1 expression with the presence of small intratumoral blood vessels, tissue sections were stained for CD31 and ETS-1. Lower ETS-1 reactivity was observed in hypercellular tumor areas with low microvessel density (Figure 2a and b), whereas enhanced ETS-1-expression was found in hypercellular tumor areas with high CD31-positive microvessel density (Figure $2 \mathrm{c}$ and $\mathrm{d}$ ). One of the examined tumors (TB 771) showed an increased number of glioma cells with a positive ETS-1 signal alongside larger arterial and venous vessels (arrow in Figure 1c).

Expression of ETS-1 in human GBM cells. To further characterize the expression of ETS-1 in specific subpopulations of tumor cells, gbMSLCs isolated from ETS-
1 positive tumors, one bmMSC cell line, as well as U87, U251, and U373 were stained for ETS-1. In all tumor cell lines examined, strong specific nuclear and perinuclear reactivity for ETS-1 was found (Figures 3 and 4). Compared to bmMSCs, gbMSLCs showed a stronger staining intensity (Figure 3d).

The influence of hypoxia on the expression of ETS-1 in glioma cells. In the next step, the influence of hypoxia on ETS-1 expression in different GBM-derived cells was examined. ETS-1 mRNA expression was assessed by RT-PCR and ETS-1 protein expression by immunoblotting. Comparing the results of three independent experiments, hypoxia did not have a significant impact on ETS-1 mRNA expression in U87, U251 and U373 cells $(p \geq 0.2)$ (Figure 5a). These findings were confirmed on the protein level $(p \geq 0.4)$ (Figure $5 b)$. Analyzing the results of three independent experiments for gbMSLCs together, a slight decrease of ETS-1 mRNA expression in all gbMSLCs under hypoxic conditions was found, although these findings were not significant $(p \geq 0.1$ each) (Figure 5c). Moreover, on the protein level, there was no significant effect of hypoxia on ETS-1 expression in gbMSLCs and bmMSCs $(p \geq 0.1)$ (Figure 5d). In comparison to gbMSLCs, the ETS- 1 mRNA expression in bmMSC was relatively weaker. This was confirmed by immunoblotting (Figure 5d).

The influence of hypoxia on the expression of ETS-1 in endothelial cells. Since positive staining for ETS-1 was observed in endothelial cells of small tumor vessels in the examined tumors, the influence of hypoxia on ETS-1 expression in endothelial cells isolated from primary GBM was assessed. For comparison, HUVECs were used, as Oikawa et al. described these as expressing ETS-1 in a hypoxia-dependent manner (20). Considering previous reports that different growth factors induce ETS-1 in endothelial cells, all examined endothelial cells were incubated in serum- and growth factor-free medium under normoxic or hypoxic conditions while conducting experiments (8). No substantial influence of hypoxia on ETS1 mRNA expression was observed in any endothelial cell line examined ( $p \geq 0.7$ each) (Figure 5e). Moreover, on the protein level, there was no significant effect of hypoxia on ETS-1 expression ( $p \geq 0.4$ each) (Figure 5f).

\section{Discussion}

In different tumor entities, ETS-1 expression has been shown to increase with tumor grading and to be an independent predictive factor for an unfavorable prognosis and poor patient outcome (12-14). In accordance with these findings, Kitange et al. verified a correlation between the expression of ETS-1 and the histological grading in astrocytic tumors (15). 


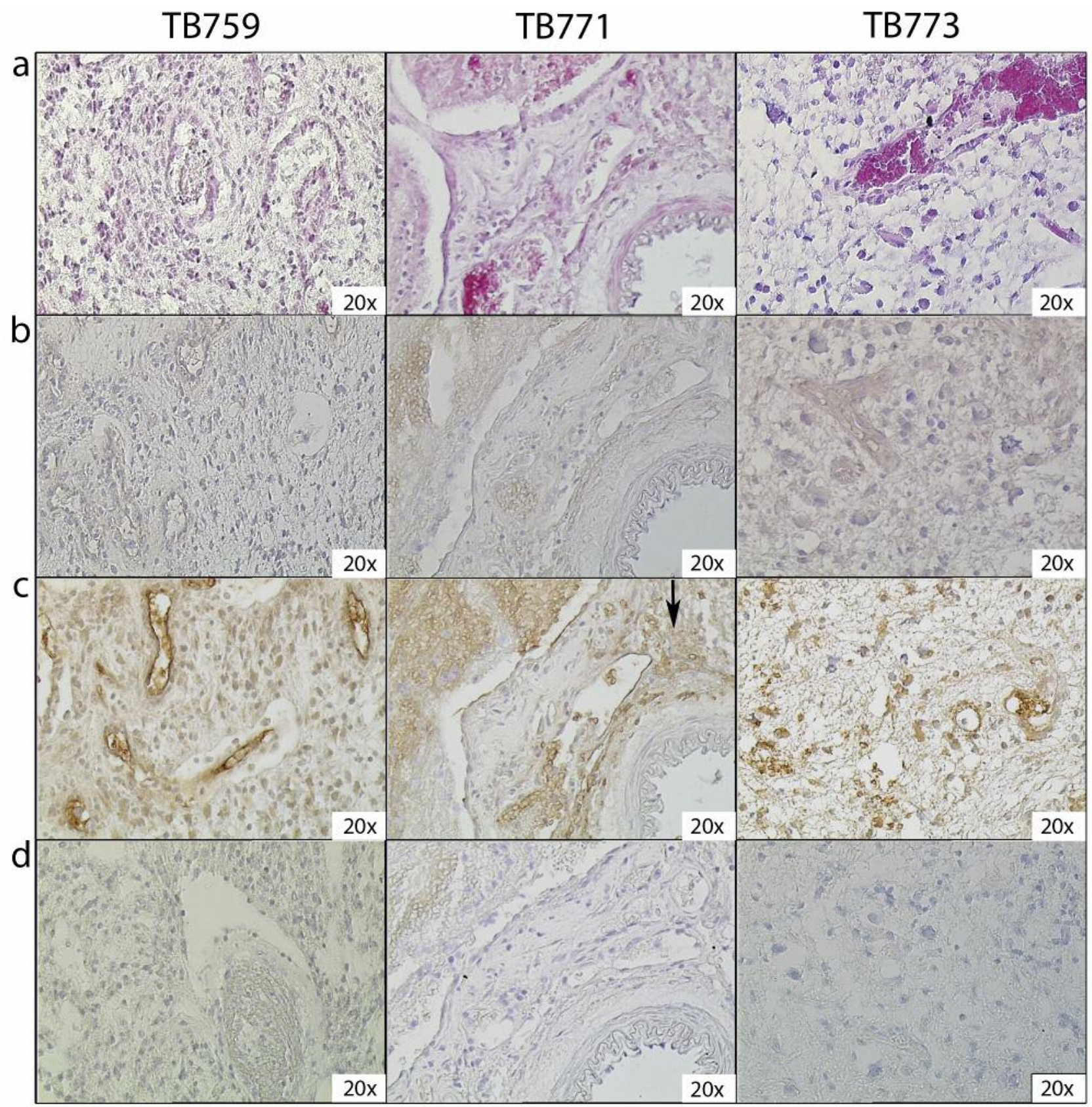

Figure 1. Identification of ETS proto-oncogene 1 (ETS-1)-positive primary glioblastoma. Three different primary glioblastoma tissues were examined: T759, TB771 and TB773. a: Hematoxylin/eosin staining. $b$ : Immunohistochemical staining for isocitratedehydrogenase 1 (IDH1) mutation: no reactivity in all three tumors. c: Immunohistochemical staining for ETS-1: specific positive reactivity in both glioma cells and endothelial cells. $d$ : Negative control. Arrow: Enhanced perivascular ETS-1 signal alongside larger blood vessels.

While reviewing the literature, discrepancies were found regarding the expression pattern of ETS-1 reported in GBM. Whereas Valter et al. described ETS-1 as being restricted to active endothelial cells (16), Kitange et al. reported ETS-1 is expressed in the endothelial cells of small vessels as well in up to $90 \%$ of the tumor cells $(15,16)$. Therefore, the first goal of this work was to elucidate the expression pattern of ETS1 in GBM. In several non-glial tumor entities, ETS-1 expression has been described to correlate with microvessel density (9). In our studies, ETS-1 was expressed in 


\section{CD31 $\quad$ ETS-1}

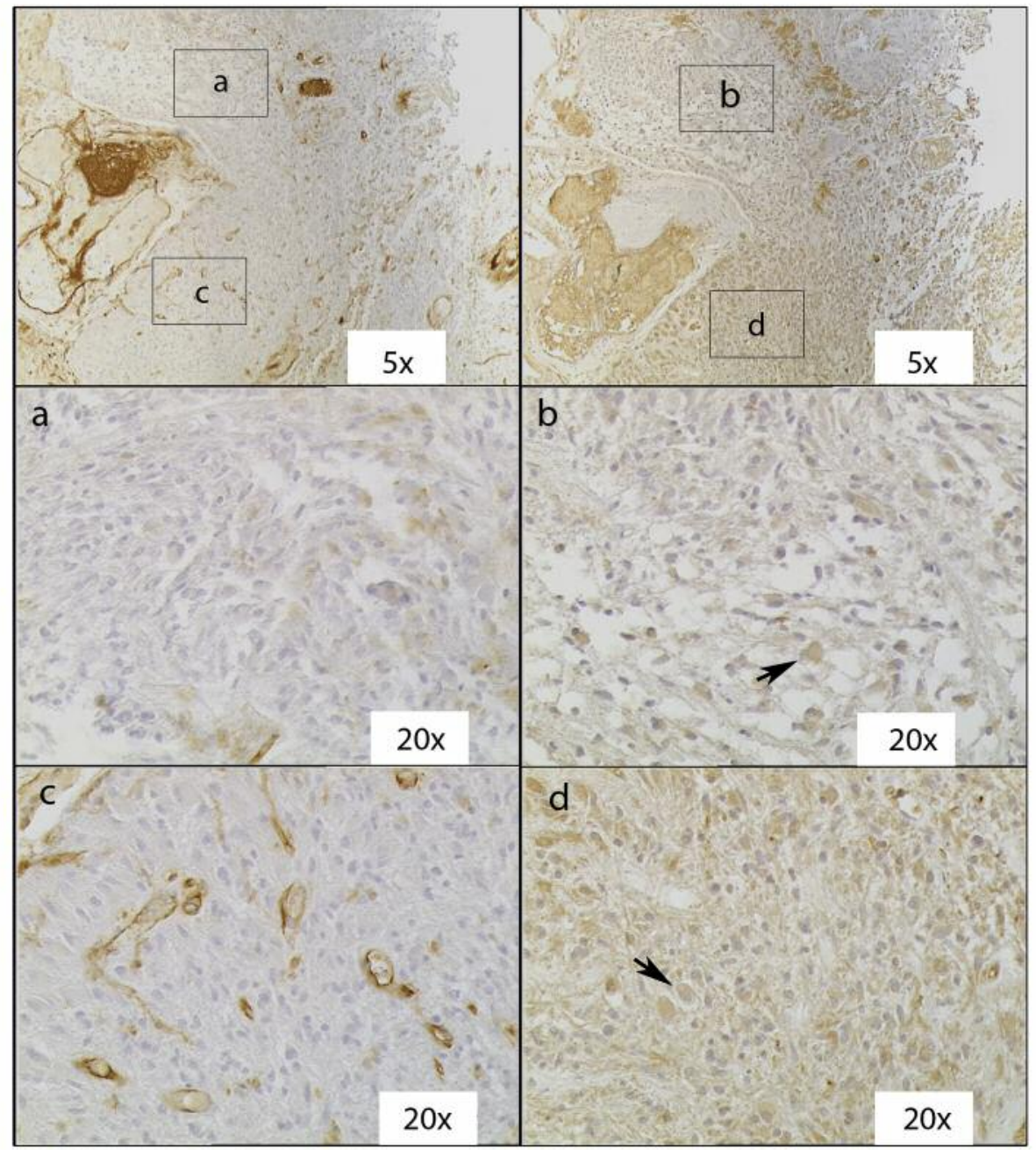

Figure 2. Correlation of CD31- and ETS proto-oncogene 1 (ETS-1) expression. a,b: Diminished ETS-1 expression in tumor areas with low microvessel density (low CD31 expression). $c$, d: Enhanced ETS-1 expression in tumor areas with high microvessel density (high CD31 expression). Arrow: Perinuclear signal of ETS-1 in glioma cells.

endothelial and tumor cells. To our knowledge for the first time, ETS-1 expression in GBM tissue was found to correlate with microvessel density (assessed by staining for endothelial cell marker CD31).

Our findings do not contradict the results of Kitange et al., who described homogenous staining in the examined tumors, as they did not correlate ETS-1 expression with microvessel density (15). The difference compared to the findings of Valter et al., who only observed ETS-1 expression in active endothelial cells, might well be explained by the histopathological heterogeneity often observed in glio-blastoma (16).

In additional immunocytochemical staining, ETS-1 expression in gbMSLCs was confirmed. They showed a strong nuclear and perinuclear signal, which was stronger compared to that of bmMSCs. The weaker expression in bmMSCs was confirmed by immunoblotting. Our results indicate that increased ETS-1 expression might be a distinctive property of gbMSLCs, in comparison to bmMSCs, indicating a potential role for ETS-1 in malignant progression of mesenchymal stem-like cells. This falls in line with our findings that in contrast to non-glial tumor cell lines, hypoxia on its own has no significant impact on ETS1 expression in glioma cells, nor in GBM-derived endothelial cells $(20,21)$.

Taking the findings from Qiao et al. into account, who demonstrated that hypoxia transiently increased ETS-1 


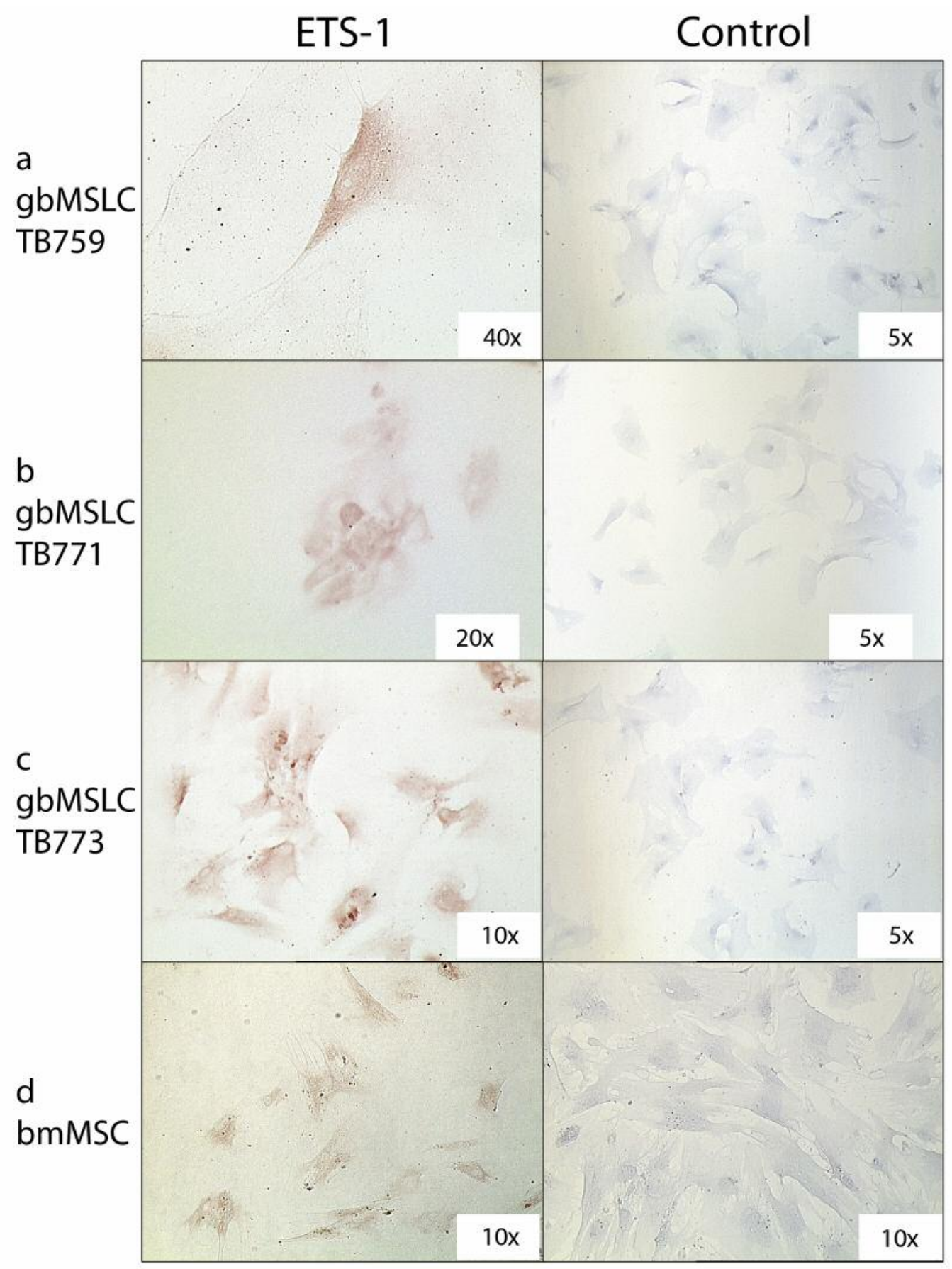

Figure 3. Immunocytochemical staining for ETS proto-oncogene 1 (ETS-1) in glioblastoma-derived mesenchymal stem-like cells (gbMSLCs). ETS-1 immunocytochemical staining in gbMSCLCs (a-c), compared to bone marrow-derived stem cells (bmMSC BM20) (d): Nuclear and perinuclear reactivity for ETS-1 in gbMSCLCs, lower staining intensity in bmMSC. Corresponding negative controls are given in the right-hand panel.

mRNA expression in pancreatic adenoma cell lines while concurrently reducing the ETS-1 protein level, our findings support that the effect of hypoxia on ETS-1 expression seems to be tissue-dependent (22).
As previous studies have shown that FBS and vascular endothelial growth factor induce ETS-1 in glioma cells and endothelial cells, we refrained from using these in our hypoxia assays $(8,18)$. Previous experiments showing the 


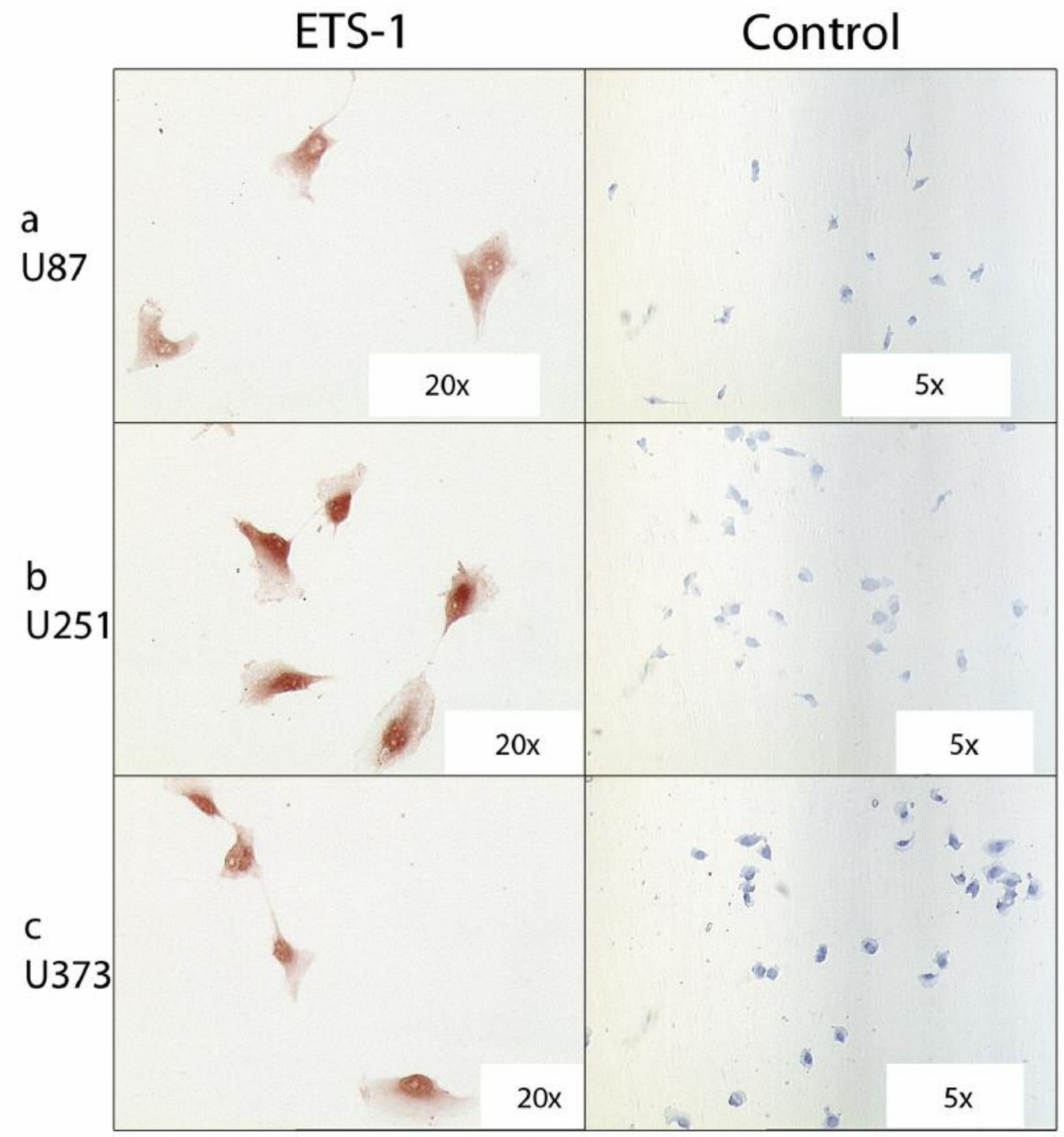

Figure 4. Immunocytochemical staining for ETS proto-oncogene 1 (ETS-1) in glioblastoma cell lines. a-c: ETS-1 immunocytochemical staining in glioblastoma cell lines: nuclear and perinuclear reactivity for ETS-1. Corresponding negative controls are given in the right-hand panel.

induction of ETS-1-expression by hypoxia in carcinoma cell lines and HUVECs were also conducted in FBS- or growth factor-containing media $(20,21)$. Therefore, we cannot rule out the possibility that hypoxia might potentiate the effect of FBS and different growth factors in inducing ETS-1 in glioma. This should be addressed in future experiments.

\section{Acknowledgements}

The Authors thank S. Lakotta, S. Lange and A. Niedermeier for their technical assistance. This research was supported by a grant from the Friedrich-Baur-Foundation.

\section{References}

1 Stupp R, Mason WP, van den Bent MJ, Weller M, Fisher B, Taphoorn MJ, Belanger K, Brandes AA, Marosi C, Bogdahn U, Curschmann J, Janzer RC, Ludwin SK, Gorlia T, Allgeier A, Lacombe D, Cairncross JG, Eisenhauer E and Mirimanoff RO: Radiotherapy plus concomitant and adjuvant temozolomide for glioblastoma. N Engl J Med 352(10): $987-$ 996, 2005.

2 Ostrom QT, Gittleman H, Liao P, Rouse C, Chen Y, Dowling J, Wolinsky Y, Kruchko C and Barnholtz-Sloan J: Cbtrus statistical report: Primary brain and central nervous system tumors diagnosed in the united states in 2007-2011. Neuro Oncol 16(Suppl 4): iv1-iv63, 2014. 
a
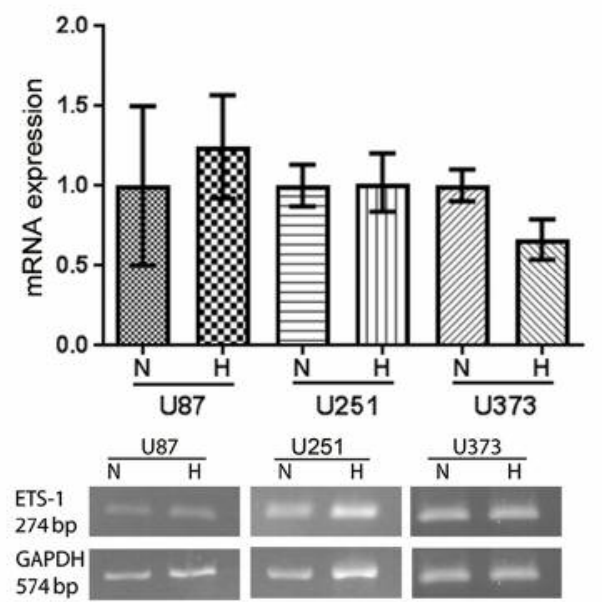

C
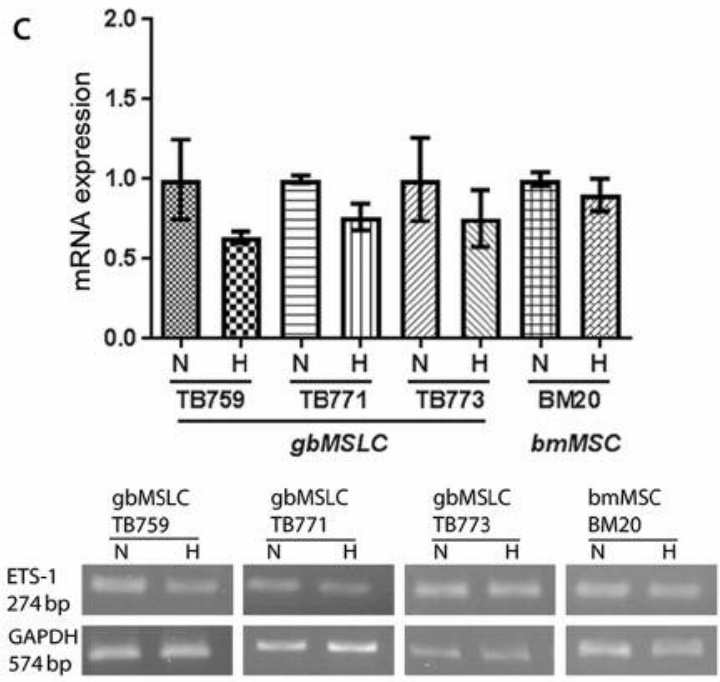

e
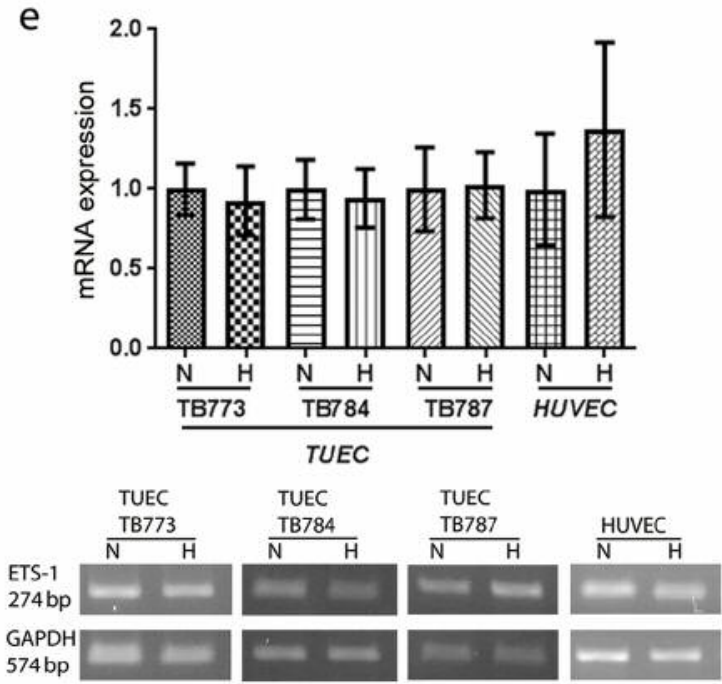

b
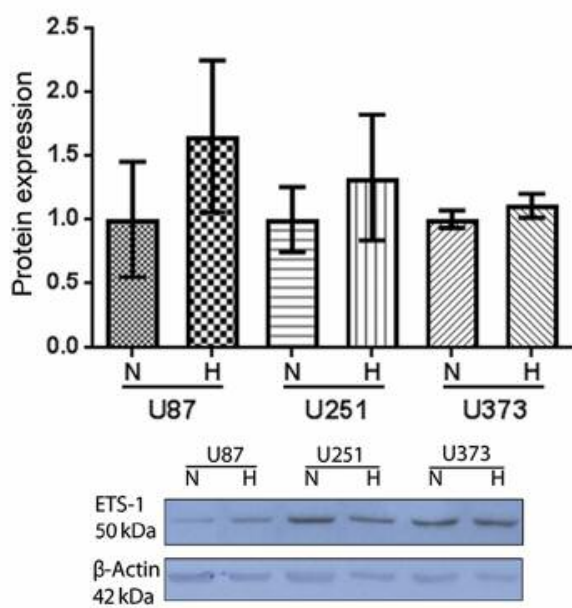

d
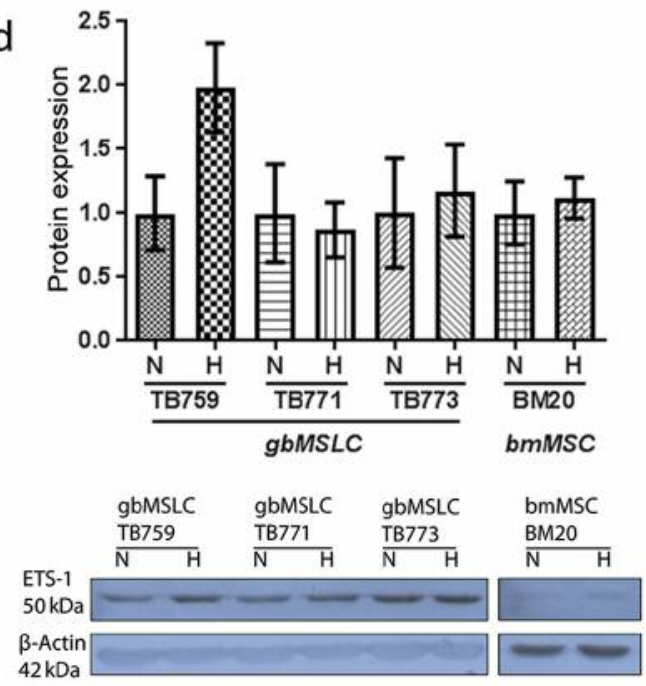

$f$
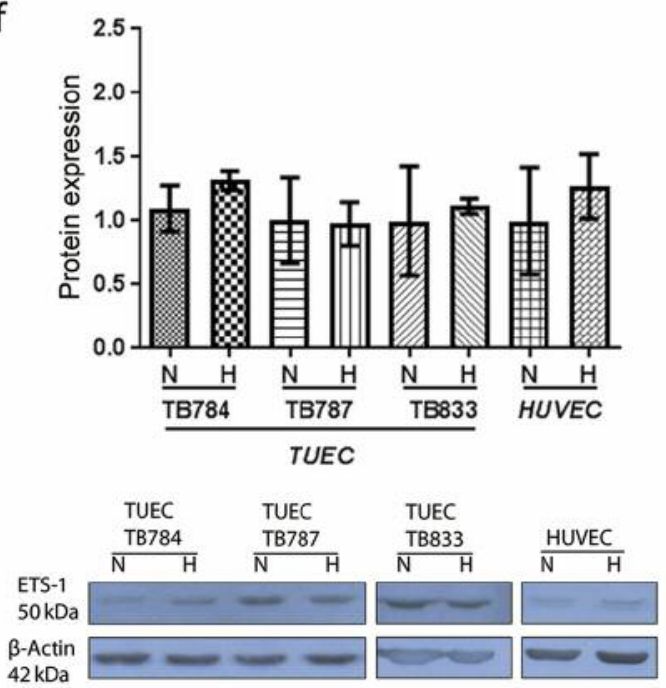

Figure 5. Hypoxia does not induce persistent changes in ETS proto-oncogene 1 (ETS-1) expression in primary glioblastoma. Relative ETS-1 mRNA and protein expression in U87, U251 and U373 cells ( $a, b)$; glioblastoma-derived mesenchymal (gbMSLCs) and bone marrow-derived (bmMSCs) stem cells $(c, d)$; and glioblastoma-derived tumor TUEC and human umbilical vein endothelial cells (HUVEC) $(e, f)$ under 48 hormoxia (N) vs. hypoxia $(H)$. There was no significant impact of hypoxia on ETS-1 mRNA in any of the assays. All values are the mean of three independent experiments \pm SEM. 
3 Bao S, Wu Q, McLendon RE, Hao Y, Shi Q, Hjelmeland AB, Dewhirst MW, Bigner DD and Rich JN: Glioma stem cells promote radioresistance by preferential activation of the DNA damage response. Nature 444(7120): 756-760, 2006.

4 Leprince D, Gegonne A, Coll J, de Taisne C, Schneeberger A, Lagrou C and Stehelin D: A putative second cell-derived oncogene of the avian leukaemia retrovirus e26. Nature 306(5941): 395-397, 1983

5 Nunn MF, Seeburg PH, Moscovici C and Duesberg PH: Tripartite structure of the avian erythroblastosis virus e26 transforming gene. Nature 306(5941): 391-395, 1983.

6 Anderson MK, Hernandez-Hoyos G, Diamond RA and Rothenberg EV: Precise developmental regulation of ets family transcription factors during specification and commitment to the T-cell lineage. Development 126(14): 3131-3148, 1999.

7 Clausen PA, Athanasiou M, Chen Z, Dunn KJ, Zhang Q, Lautenberger JA, Mavrothalassitis G and Blair DG: ETS-1 induces increased expression of erythroid markers in the pluripotent erythroleukemic cell lines K562 and HEL. Leukemia 11(8): 1224-1233, 1997.

8 Iwasaka $\mathrm{C}$, Tanaka $\mathrm{K}$, Abe $\mathrm{M}$ and Sato $\mathrm{Y}$ : Ets-1 regulates angiogenesis by inducing the expression of urokinase-type plasminogen activator and matrix metalloproteinase- 1 and the migration of vascular endothelial cells. J Cell Physiol 169(3): 522-531, 1996.

9 Dittmer J: The biology of the ETS1 proto-oncogene. Mol Cancer 2: $29,2003$.

10 Hahne JC, Okuducu AF, Sahin A, Fafeur V, Kiriakidis S and Wernert N: The transcription factor ETS-1: Its role in tumour development and strategies for its inhibition. Mini Rev Med Chem 8(11): 1095-1105, 2008.

11 Kim E, Gunther W, Yoshizato K, Meissner H, Zapf S, Nusing RM, Yamamoto H, Van Meir EG, Deppert W and Giese A: Tumor suppressor p53 inhibits transcriptional activation of invasion gene thromboxane synthase mediated by the protooncogenic factor ETS-1. Oncogene 22(49): 7716-7727, 2003.

12 Span PN, Manders P, Heuvel JJ, Thomas CM, Bosch RR, Beex LV and Sweep CG: Expression of the transcription factor ETS1 is an independent prognostic marker for relapse-free survival in breast cancer. Oncogene 21(55): 8506-8509, 2002.

13 Davidson B, Reich R, Goldberg I, Gotlieb WH, Kopolovic J, Berner A, Ben-Baruch G, Bryne M and Nesland JM: ETS-1 messenger rna expression is a novel marker of poor survival in ovarian carcinoma. Clin Cancer Res 7(3): 551-557, 2001.

14 Duda DG, Sunamura M, Lefter LP, Furukawa T, Yokoyama T, Yatsuoka T, Abe T, Inoue H, Motoi F, Egawa S, Matsuno S and Horii A: Restoration of SMAD4 by gene therapy reverses the invasive phenotype in pancreatic adenocarcinoma cells. Oncogene 22(44): 6857-6864, 2003.

15 Kitange G, Kishikawa M, Nakayama T, Naito S, Iseki M and Shibata S: Expression of the ETS-1 proto-oncogene correlates with malignant potential in human astrocytic tumors. Mod Pathol 12(6): 618-626, 1999.
16 Valter MM, Hugel A, Huang HJ, Cavenee WK, Wiestler OD, Pietsch T and Wernert N: Expression of the ETS-1 transcription factor in human astrocytomas is associated with fms-like tyrosine kinase-1 (FLT-1)/vascular endothelial growth factor receptor-1 synthesis and neoangiogenesis. Cancer Res 59(21): 5608-5614, 1999.

17 Nakada M, Yamashita J, Okada Y and Sato H: Ets-1 positively regulates expression of urokinase-type plasminogen activator (UPA) and invasiveness of astrocytic tumors. J Neuropathol Exp Neurol 58(4): 329-334, 1999.

18 Kitange G, Shibata S, Tokunaga Y, Yagi N, Yasunaga A, Kishikawa $\mathrm{M}$ and Naito S: Ets-1 transcription factor-mediated urokinase-type plasminogen activator expression and invasion in glioma cells stimulated by serum and basic fibroblast growth factors. Lab Invest 79(4): 407-416, 1999.

19 Sahin A, Vercamer C, Kaminski A, Fuchs T, Florin A, Hahne JC, Mattot V, Pourtier-Manzanedo A, Pietsch T, Fafeur V and Wernert N: Dominant-negative inhibition of ETS-1 suppresses tumor growth, invasion and migration in rat c6 glioma cells and reveals differentially expressed ETS-1 target genes. Int J Oncol 34(2): 377-389, 2009.

20 Oikawa M, Abe M, Kurosawa H, Hida W, Shirato K and Sato Y: Hypoxia induces transcription factor ETS-1 via the activity of hypoxia-inducible factor-1. Biochem Biophys Res Commun 289(1): 39-43, 2001.

21 Mamori S and Tajiri H: ETS-1 is increased in anticancer drugcontaining media and hypoxic cultures, similar to TACE. Scand J Gastroenterol 44(4): 507-508, 2009.

22 Qiao N, Xu C, Zhu YX, Cao Y, Liu DC and Han X: ETS-1 as an early response gene against hypoxia-induced apoptosis in pancreatic beta-cells. Cell Death Dis 6: e1650, 2015.

23 Louis DN, Perry A, Reifenberger G, von Deimling A, FigarellaBranger D, Cavenee WK, Ohgaki H, Wiestler OD, Kleihues P and Ellison DW: The 2016 World Health Organization Classification of Tumors of the Central Nervous System: A summary. Acta Neuropathol 131(6): 803-820, 2016.

24 Schichor C, Albrecht V, Korte B, Buchner A, Riesenberg R, Mysliwietz J, Paron I, Motaln H, Turnsek TL, Jurchott K, Selbig $\mathrm{J}$ and Tonn JC: Mesenchymal stem cells and glioma cells form a structural as well as a functional syncytium in vitro. Exp Neurol 234(1): 208-219, 2012.

25 Guo KT, Fu P, Juerchott K, Motaln H, Selbig J, Lah T, Tonn JC and Schichor C: The expression of WNT-inhibitor DKK1 (dickkopf 1) is determined by intercellular crosstalk and hypoxia in human malignant gliomas. J Cancer Res Clin Oncol 140(8): 1261-1270, 2014.

26 Schneider CA, Rasband WS and Eliceiri KW: NIH image to ImageJ: 25 years of image analysis. Nat Meth 9: 671, 2012.

Received March 29, 2018

Revised April 29, 2018

Accepted May 3, 2018 